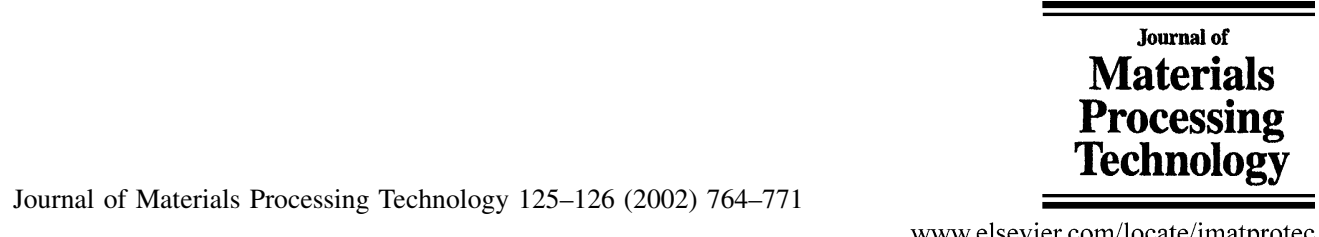

www.elsevier.com/locate/jmatprotec

\title{
Experimental and numerical study of reverse re-drawing of anisotropic sheet metals
}

\author{
S. Thuillier ${ }^{\mathrm{a},{ }^{*}, \text { P.Y. Manach }}{ }^{\mathrm{a}}$, L.F. Menezes ${ }^{\mathrm{b}}$, M.C. Oliveira ${ }^{\mathrm{b}}$ \\ ${ }^{\mathrm{a}}$ Laboratoire Génie Mécanique et Matériaux, Université de Bretagne Sud, Rue de Saint Maudé, BP 92116, 56321 Lorient Cedex, France \\ ${ }^{\mathrm{b}}$ Departamento de Engenharia Mecânica, Centro de Engenharia Mecânica da Universidade de Coimbra, \\ Universidade de Coimbra, Pinhal de Marrocos, 3030 Coimbra, Portugal
}

Received 13 December 2001; accepted 24 January 2002

\begin{abstract}
This study deals with the experimental and numerical reverse re-drawing of cylindrical cups. Experiments were carried out on a classical tensile test machine of maximum load $100 \mathrm{kN}$. Experimental data consist of force-displacement curves of the punch and thickness distribution in the cup wall at $0^{\circ}, 45^{\circ}$ and $90^{\circ}$ to the rolling direction (RD). The drawing process was simulated using both the dynamic explicit finite element code Pam-Stamp and the static implicit home code DD3IMP. Two extreme cases have been considered: a dynamic explicit calculation with shell elements, leading to low CPU times and a static implicit calculation with solid elements, which is CPU time-consuming. The accuracy of these numerical results, when compared to experimental ones, is then studied. Moreover, the occurrence of strain path changes during the first and the second stage is also investigated in order to estimate their influence.
\end{abstract}

(C) 2002 Elsevier Science B.V. All rights reserved.

Keywords: Deep drawing; Sheet metal; Experiments; Finite element simulations; Implicit/explicit calculations

\section{Introduction}

A lot of work has been dedicated to the mechanical modelling and the numerical simulation of deep drawing processes and nowadays there are several finite element codes that can deal successfully with the simulation of such processes, e.g. [1]. The main framework of the modelling is large elasto-plastic transformations, contact with Coulomb friction, orthotropic yield criterion and static implicit or dynamic explicit calculations. However, within this framework, several aspects can still be improved in order to decrease the discrepancy between experimental and numerical results. When considering multi-stage drawing, the task is even more difficult because the stress and thickness distribution resulting from the first stage will influence the subsequent behaviour. Moreover, strain path changes are expected to occur, requiring more complex constitutive laws [2].

When high drawing ratios are required, the process is decomposed into two or several steps, in order to increase the formability by preventing localisation of the deformation in the cup wall $[3,4]$. Re-drawing processes are usually

\footnotetext{
${ }^{*}$ Corresponding author. Tel.: +33-297874537; fax: +33-297874572. E-mail address: sandrine.thuillier@univ-ubs.fr (S. Thuillier).
}

sorted out in two categories: direct and reverse re-drawing [5]. The first one corresponds to a process in which the different punches are always in contact with the same blank side whereas during reverse re-drawing, the punch travel occurs in two opposite directions and the outside of the part during the first stage becomes the inside of the part in the second stage. The advantages of the reverse process are a more compact tooling, without new positioning of the part in-between the two stages, a better surface aspect than in the case of a direct process because the outside is in contact only once with the die radius and finally a smaller number of bending-unbending operations [6,7]. Previous numerical studies have investigated the influence of kinematic hardening and technological parameters as well as the comparison between direct and reverse re-drawing $[2,3,6,8]$. Axi-symmetric formulations are often used for time-saving reasons and only normal anisotropy is taken into account. It should be noticed that $3 \mathrm{D}$ simulations were mainly performed with shell elements [1].

The drawing process considered in this study is a benchmark of Numisheet'99 [9]. It consists of drawing cylindrical cups in two stages: the drawing ratio is 1.7 in the first stage and 1.36 in the second stage (to be compared with 2.32 in a single stage process). The experimental device was designed in order to be settled on a classical electromechanical tensile 


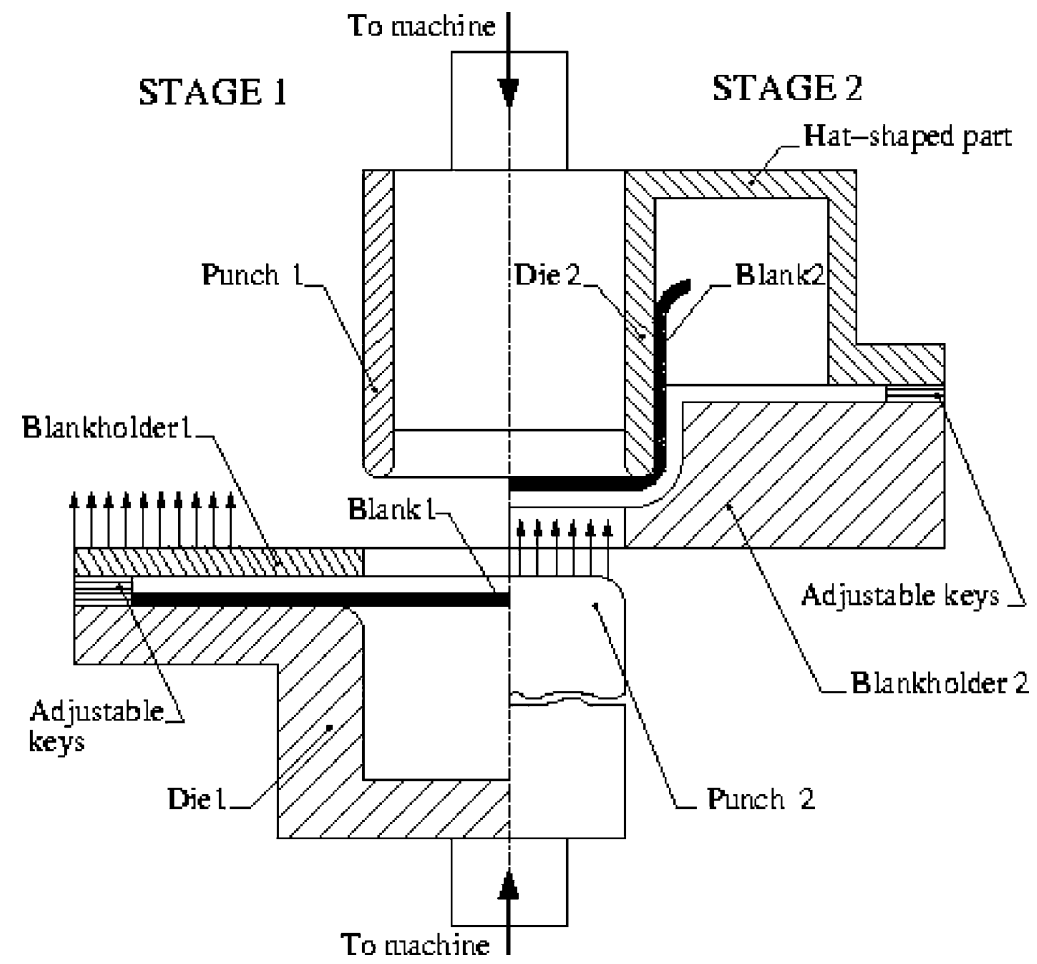

Fig. 1. Schematic representation of the deep drawing device for stage 1 (left-hand side) and stage 2 (right-hand side).

test machine of maximum capacity $100 \mathrm{kN}$. Experimental data consist of force-displacement curves of the punch and thickness distribution in the cup wall at $0^{\circ}, 45^{\circ}$ and $90^{\circ}$ to the RD. Numerical simulations were carried out with both the dynamic explicit finite element code Pam-Stamp and the static implicit home code DD3IMP [10]. Results are also compared with the results issued from the Numisheet'99 benchmark.

\section{Experimental procedure}

The device designed to perform the reverse re-drawing is presented in detail in [11] (see Fig. 1). The tool geometry for the two stages is given in Table 1. The gap between the draw die and the blank-holder was kept constant, via adjustable keys, for the two stages. The value of the gaps was determined as large as possible in order to draw a cup without

Table 1

Tool geometry for both stages (in $\mathrm{mm}$ )

\begin{tabular}{lcc}
\hline & Stage 1 & Stage 2 \\
\hline Blank: diameter, thickness & $170.0,0.98$ & - \\
Die opening diameter & 104.5 & 78.0 \\
Die radius & 8.0 & 5.5 \\
Punch diameter & 100.0 & 73.4 \\
Punch radius & 5.5 & 8.5 \\
Blank-holder opening diameter & 104.5 & 75.0 \\
Blank-holder radius & - & 7.0 \\
Gap die/blank-holder & 1.0 & 1.4 \\
\hline
\end{tabular}

wrinkles. Figs. 2 and 3 show the evolution of the punch force during the first and second stages respectively and Fig. 4 exhibits the thickness of the cup wall at $0^{\circ}, 45^{\circ}$ and $90^{\circ}$ to the $\mathrm{RD}$. The cups were drawn to $50 \mathrm{~mm}$ in stage 1 and $70 \mathrm{~mm}$ in stage 2. Blanks were lubricated (Fuchs $4107 \mathrm{~S}$ ) at the beginning of the process and also before the second stage on both sides. It can be seen in Fig. 3 that there exists an anomalous peak on the punch force-displacement curve of stage 2 . It can be associated with the passage of the thickened zone of

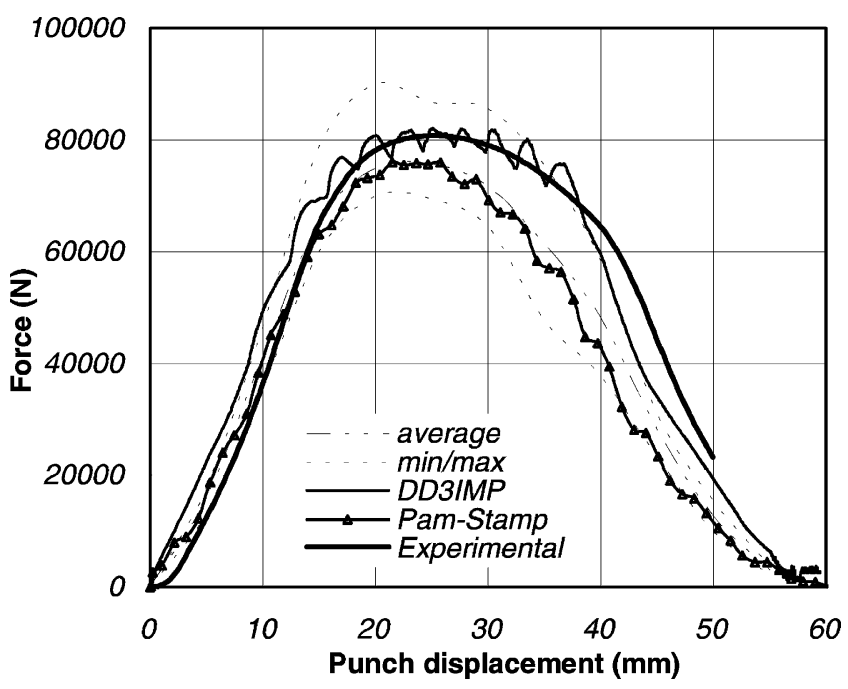

Fig. 2. Experimental and numerical punch force-displacement curves for stage 1. Average and minimum and maximum curves calculated from [1] are also plotted. 


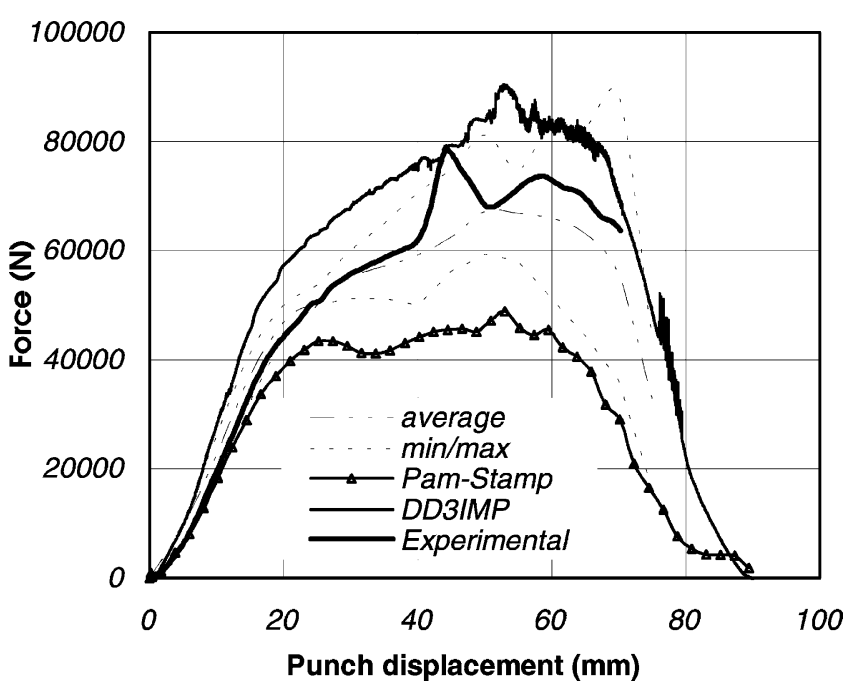

Fig. 3. Experimental and numerical punch force-displacement curves for stage 2. Average and minimum and maximum curves calculated from [1] are also plotted.
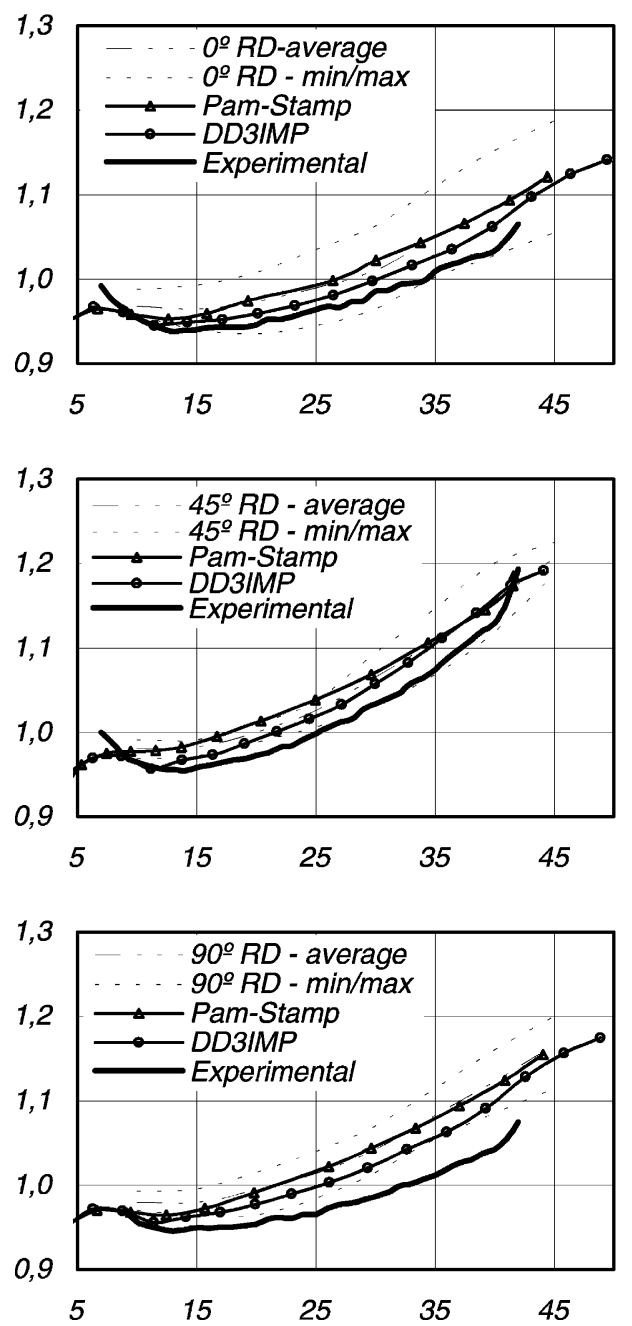

the cup rim after stage 1, between the die and the blankholder. Such a trend is also recorded by other benchmark participants but with a much lower magnitude for aluminium and mild steel. In any case, a similar peak is recorded for the dual-phase steel [1]. Moreover, such an effect is also reported in [8], in the case of drawing and re-drawing of aluminium cups.

The decomposition into two or more stages becomes necessary when high drawing ratios are required. Indeed, in the case of a single step process, a large amount of material lies under the blank-holder that leads to high restraining forces. As illustrated in Fig. 5, the single step drawing is associated to the deformation path such that an excessive thinning occurs in the cup wall. The punch force increases dramatically, i.e. $1200 \mathrm{kN}$ for a displacement of $45 \mathrm{~mm}$ (the maximum force reached during the reverse process is around $80 \mathrm{kN}$ ), until a severe strain localisation is reached. The decrease of the drawing ratio obtained with a two-stage process leads to a deformation front close to the direction $\varepsilon_{1}=-\varepsilon_{2}$, and during the second stage this front expands mainly along the same direction.
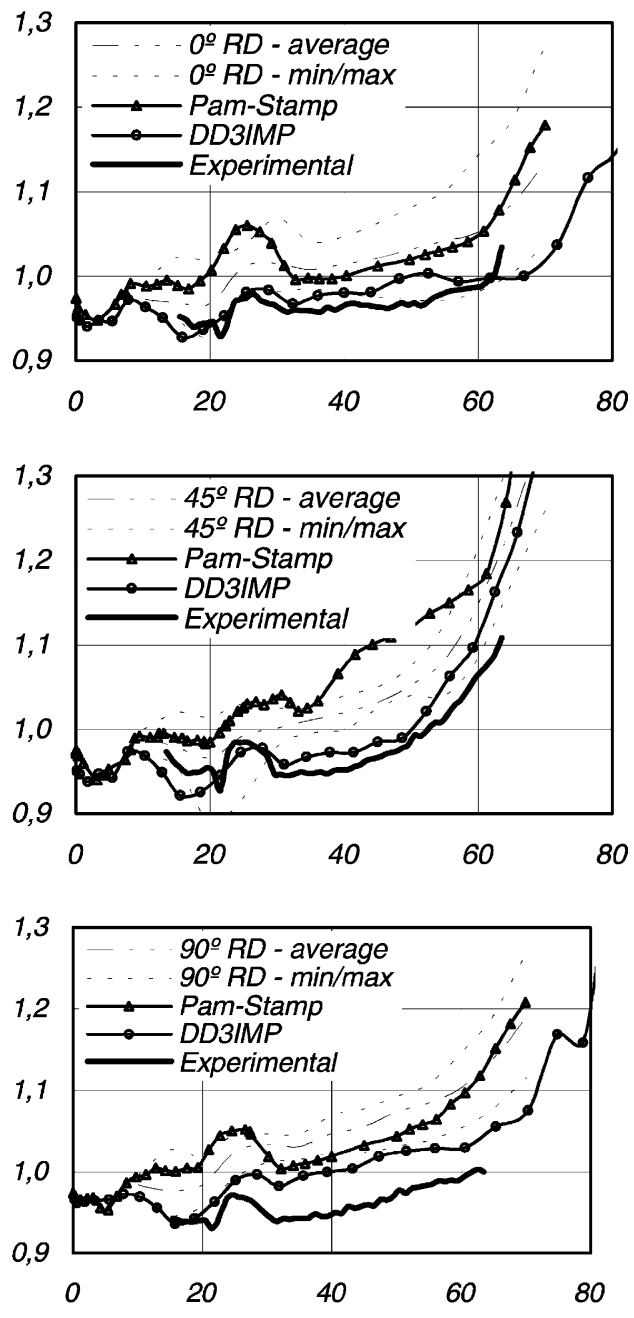

Fig. 4. Experimental and numerical thickness distribution in the cup wall after stage 1 (left-hand side) and stage 2 (right-hand side), at $0^{\circ}, 45^{\circ}$ and $90^{\circ}$ to the RD. 


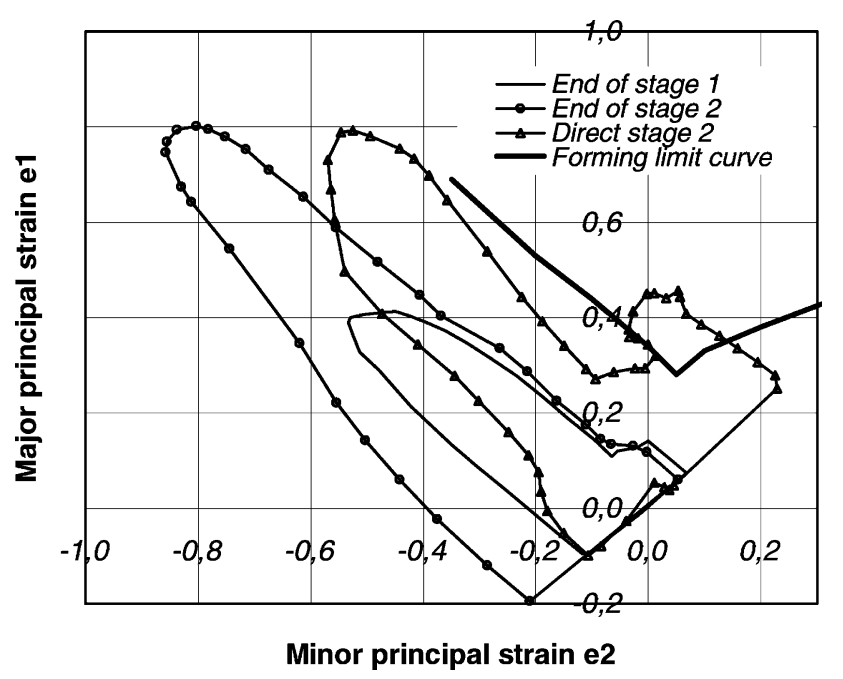

Fig. 5. Comparison of minor-major strain plots during the reverse redrawing and the equivalent single stage process. Reverse re-drawing leads to deformation path where the thickness strain is lower. Calculations are performed with DD3IMP.

\section{Constitutive behaviour}

The present study was carried out with a deep drawing quality mild steel that exhibits an anisotropic behaviour, with an average strain ratio $\bar{r}=1.55$ and a strain ratio variation $\Delta r=0.65$. The general framework of the mechanical model is small elastic strains $(E=200 \mathrm{GPa}, n=0.3)$ and finite plastic deformations. The orthotropy of the rolled metallic sheet is described with the Hill's (1948) quadratic criterion, which is

$$
\begin{aligned}
\bar{\sigma}^{2}= & F\left(\sigma_{22}-\sigma_{33}\right)^{2}+G\left(\sigma_{33}-\sigma_{11}\right)^{2}+H\left(\sigma_{11}-\sigma_{22}\right)^{2} \\
& +2 L \sigma_{23}^{2}+2 M \sigma_{31}^{2}+2 N \sigma_{12}^{2}
\end{aligned}
$$

where $\bar{\sigma}$ is the equivalent stress, and 1,2,3 stand for the rolling, transverse and normal directions, respectively. The Hill's coefficients are usually calculated from the $r$ ratios $r_{0}$, $r_{45}$ and $r_{90}$, which are the ratios of the width-to-thickness incremental plastic strain during a tensile test, at $0^{\circ}, 45^{\circ}$ and $90^{\circ}$, respectively, to the RD:

$$
\begin{array}{ll}
G=\frac{1}{1+r_{0}}, \quad H=r_{0} G, & F=\frac{G r_{0}}{r_{90}}, \\
N=G\left(\frac{r_{0}}{r_{90}}+1\right)\left(r_{45}+0.5\right), & L=M=N
\end{array}
$$

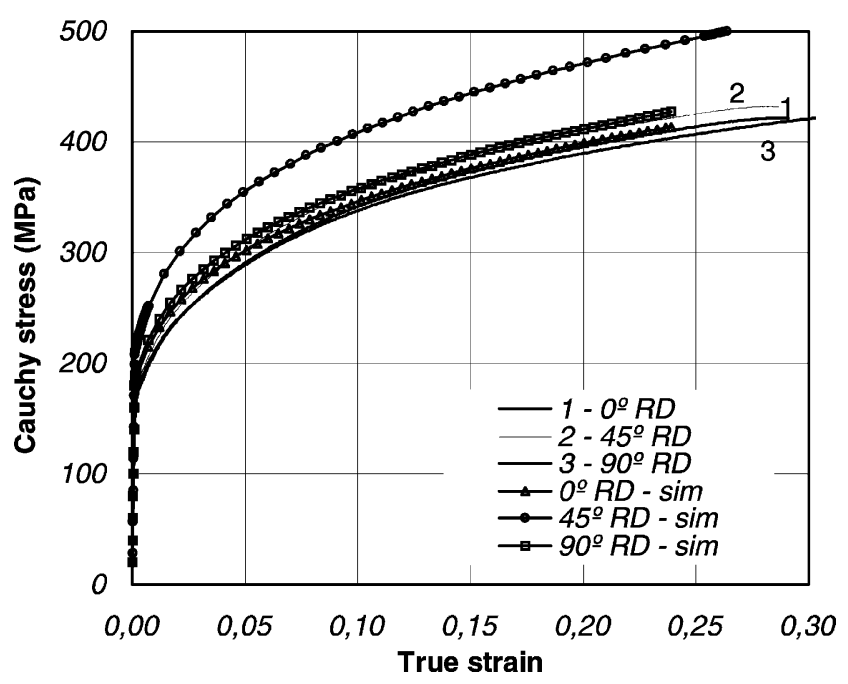

Fig. 6. Experimental and simulated tensile test data. Numerical results are obtained using material parameter set 1 . The use of set 2 gives similar results.

The isotropic hardening is described by a Swift law: $\bar{\sigma}=K\left(\varepsilon_{0}+\bar{\varepsilon}^{\mathrm{P}}\right)^{n}$ where $\bar{\varepsilon}^{\mathrm{P}}$ is the equivalent plastic strain, $\varepsilon_{0}=\left(\sigma_{0} / K\right)^{1 / n}$, and $K, n, \sigma_{0}$ are material parameters. Corresponding material parameters were given in the benchmark specifications and are shown in Table 2 (set 1). As uniaxial tensile data were given, material parameters have also been identified directly from stress-strain curves (set 2).

With either set 1 or set 2 , tensile tests at $0^{\circ}, 45^{\circ}$ and $90^{\circ}$ to the $\mathrm{RD}$ are performed numerically and compared with experimental curves (Fig. 6). It can be seen that the description is satisfying in the RD, especially for strains above 0.1 . But at $45^{\circ}$ and $90^{\circ}$ to the $\mathrm{RD}$, the simulated curves are quite far from experimental ones. Indeed, the experimental curve at $90^{\circ}$ is slightly below the one at $0^{\circ}$ but the simulated one lies above it. At $45^{\circ}$ to the $\mathrm{RD}$, the simulated curve lies around $50 \mathrm{MPa}$ above the experimental curve. Therefore, the modelled material has a higher strength at $45^{\circ}$ and $90^{\circ}$ to the $\mathrm{RD}$ than the real material. Starting from this discrepancy, another identification is tried by fitting directly the Hill's coefficients in order to minimise the gap between experimental and simulated curves in the three directions (Table 2, set 3). In this way, tensile data in the three directions are closely fitted. It should be noticed that the coefficient $N$ becomes then very close to 1.5 , which is its value for

Table 2

Material parameters for mild steel ${ }^{\mathrm{a}}$

\begin{tabular}{lllllllllll}
\hline & $\sigma_{0}(\mathrm{MPa})$ & $K(\mathrm{MPa})$ & $n$ & $r_{0}$ & $r_{45}$ & $r_{90}$ & $F$ & $G$ & $H$ \\
\hline Set 1 & 180.3 & 555.3 & 0.208 & 1.73 & 1.23 & 2.02 & 0.314 & 0.366 & 0.634 \\
Set 2 & 155.0 & 556.1 & 0.210 & 1.814 & 1.286 & 2.10 & 0.307 & 0.355 & 0.645 \\
Set 3 & 155.0 & 556.1 & 0.210 & 1.56 & 1.31 & 1.42 & 0.430 & 0.391 & 0.609 & 1.183 \\
\hline
\end{tabular}

${ }^{a}$ Set 1: benchmark data; set 2: parameters identified from stress-strain curves; set 3: Hill's coefficients determined by fitting the stress-strain curves. 

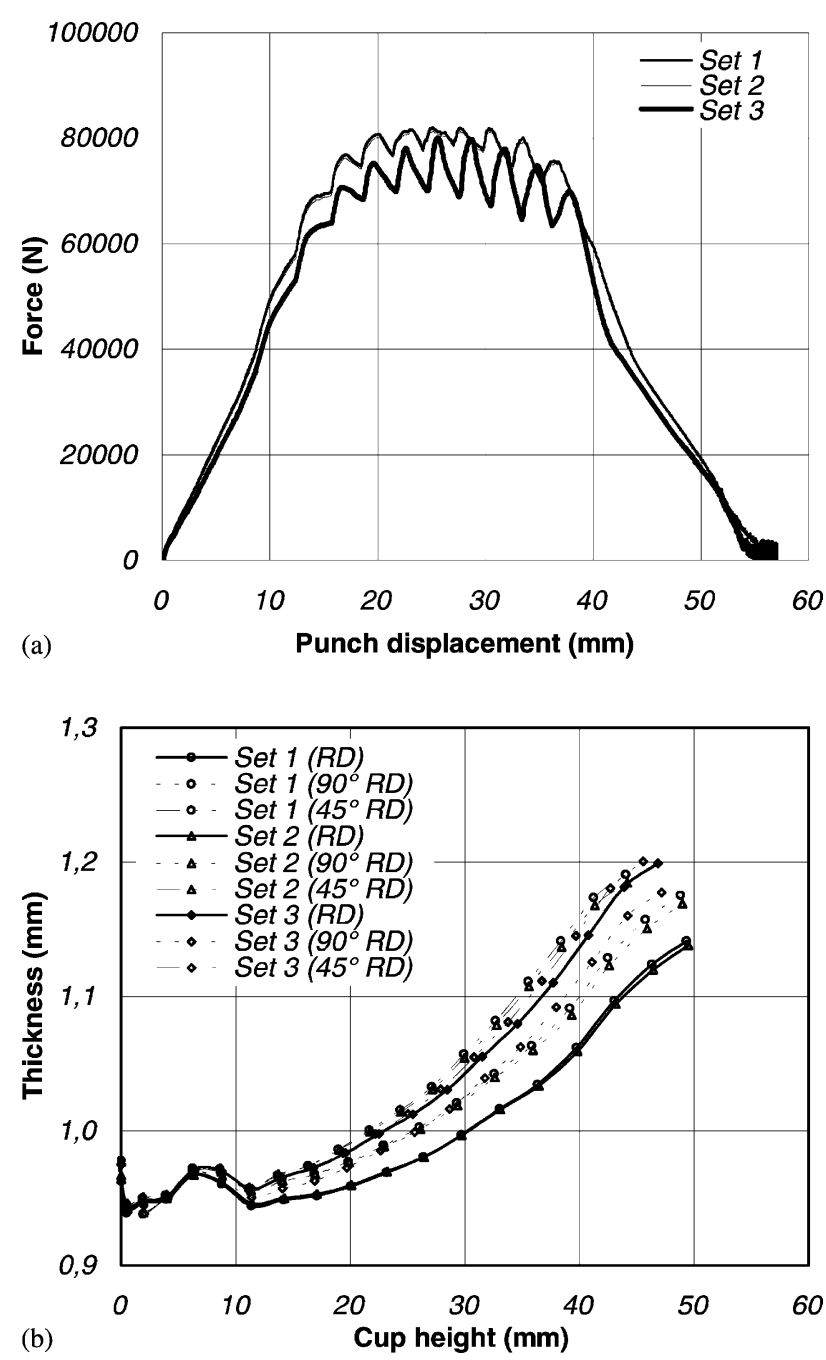

Fig. 7. Punch load-displacement curve (a) and thickness evolution at $0^{\circ}$, $45^{\circ}$ and $90^{\circ}$ to the $\mathrm{RD}$ (b) for the first stage, using the three sets of material parameters. Load-displacement curves obtained with sets 1 and 2 are almost the same.

isotropic behaviour. Numerical simulations of the first stage are performed with these three sets of parameters and results are displayed in Fig. 7. A variation of the yield strength from 155 to $180 \mathrm{MPa}$ as well as small variations of $r$ ratios do not alter significantly either the force-displacement curve or the thickness distribution. But Hill's coefficients identified from stress-strain curves lead to a decrease of the force-displacement curve (around 7\%) and influence greatly the thickness evolution. From these results, it seems that thickness at different orientations to the RD is controlled mainly by the flow stress in that direction. Indeed, thickness at a given orientation is all the more higher as the flow stress in the corresponding direction is high. As will be shown later, experimental results at $0^{\circ}$ and $90^{\circ}$ to the RD are very close and lower than thickness at $45^{\circ}$ to the RD. It seems therefore that both the flow stress and the $r$ ratio values control the thickness, in an uncoupled way.
It can be concluded from numerical tensile test results, that the mechanical model does not lead to a correct prediction of the tensile behaviour at $45^{\circ}$ and $90^{\circ}$ to the RD. This fact involves not only the Hill's criterion but also the assumption of isotropic hardening and normality rule. This assumption is known to be questionable, e.g. [12], but this model is still widely used. A previous study dealing with a different drawing process [13] showed also the result sensitivity to the determination of Hill's coefficients. Using this anisotropic yield criterion, it is not possible to fit correctly both the stress-strain curves in tension and the $r$ ratios. Either one set of data or the other can be fitted. Such a problem can be overcome by defining a criterion depending on more independent parameters, in order to fit all the data [14].

The friction coefficient is also taken from the benchmark specifications, $\mu=0.15$ in the lubricated case. Though already used in previous studies dealing with comparisons between experimental and numerical results $[3,15]$, the friction coefficient here is not an adjustable parameter to fit the experimental data.

\section{Numerical simulation of reverse re-drawing}

The drawing process was simulated using both the dynamic explicit finite element code Pam-Stamp and the static implicit home code DD3IMP. ${ }^{1}$ Concerning DD3IMP, the 3D kinematic description naturally takes into account shear and bending effects, and uses an updated Lagrangian scheme. Elastic strains are assumed negligibly small with respect to unity but large rotations are taken into account. The Coulomb law describes the contact with friction and its evolution is controlled by a mixed formulation (kinematic and static), using an augmented Lagrangian approach. Contact between a deformable sheet and rigid tools is modelled with Bézier surfaces $[10,16]$.

There have been, e.g. $[15,17]$, numerous studies on the comparison between explicit and implicit calculations of forming processes, and between the use of shell or solid elements. There is a general agreement that explicit calculations are more rapid, but when using high speed, inertial effects can occur. Conversely, the use of solid elements gives a better calculation of the stress gradients in the thickness. However, static implicit calculation with solid elements is CPU time-consuming. The characteristics of each calculation are indicated in Table 3. Deformed meshes obtained with both finite element codes are shown in Fig. 8.

The average and minimum and maximum curves calculated from the results of the Numisheet'99 results conference [1] are also plotted in Figs. 2 and 3. Out of seven calculations, five were performed in dynamic explicit and two in static implicit. Moreover, six of them made use of shell elements and one of solid elements.

\footnotetext{
${ }^{1}$ DD3IMP: contraction of Deep Drawing 3D IMPlicit finite element code.
} 
Table 3

Characteristics of the finite element simulations

\begin{tabular}{lllll}
\hline & Speed $(\mathrm{m} / \mathrm{s})$ & Elements & Number of elements & Gap stage 1 \\
\hline Pam-Stamp & 0.2 & 4-Node shell & $3008(1 / 1)+$ refinement without de-refinement & 1.0 \\
DD3IMP & - & 8-Node hexahedrons & $2016(1 / 4)$ & 0.98 \\
\hline
\end{tabular}

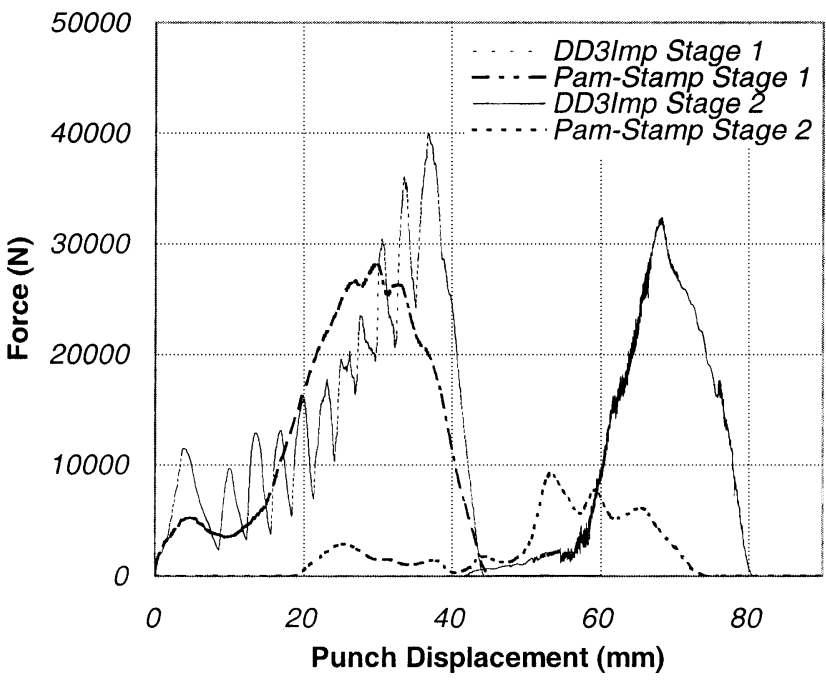

Fig. 8. Numerical cylindrical cups obtained with Pam-Stamp (left-hand side) and with DD3IMP (right-hand side). The cups in the upper parts corresponds to the end of stage 1, with a punch displacement of $60 \mathrm{~mm}$, whereas in the lower part, the cups correspond to a displacement of $60 \mathrm{~mm}$ in the reverse direction.

\section{Results and discussion}

\subsection{Stage 1}

It can be seen that the curve obtained with Pam-Stamp is very close to the average curve obtained from Numisheet' 99 results. This can come from the fact that $71 \%$ of the calculations were performed in dynamic explicit or $86 \%$ of them made use of shell elements. In all cases it seems that the constitutive law is the same. The main tendency is that the maximum force is lower than the experimental one (75.8 and $80.7 \mathrm{kN}$, respectively) and the width of the numerical curve is smaller than the experimental one (26.4 and $31.8 \mathrm{~mm}$, respectively, at $50 \mathrm{kN}$ ). The numerical simulation with DD3IMP gives a better fit to the experimental results, both in terms of the maximum force and the width of the curve $(81 \mathrm{kN}$ and $31.4 \mathrm{~mm}$, respectively). The numerical curve starts with a higher slope than the experimental one and it can be due to elastic deflection of the tools or to a false rigidity of the blank due to the linear interpolation of the finite elements. The oscillations on the DD3IMP curve are due to numerical instabilities related to contact between the deformed mesh and the tool. Indeed, when using a concentric mesh, all the elements at a given radius will come in contact with the tool at the same time. The occurrence of such instabilities is linked to the ratio of mesh element size and curvature radius of the tools. To avoid such problems, the mesh size should be lower than $20 \%$ of the curvature radius of the die, that is $1.6 \mathrm{~mm}$ for the first stage (the actual mesh size is around $3 \mathrm{~mm}$ ). But such a small element size will lead to prohibitive time for calculation. The difference in the width of the curve between Pam-Stamp and DD3IMP may come from the force applied on the blank-holder (Fig. 9). The shape is similar but with Pam-Stamp the force decreases more rapidly than with DD3IMP after a displacement of 35-40 mm.

Finally, thickness distributions lie within $10 \%$ of the experimental data and results obtained with DD3IMP are closer to experimental results in the three directions considered (around 4-5\%). However, thickness at $45^{\circ}$ and $90^{\circ}$ to the $\mathrm{RD}$ is overestimated.

\subsection{Stage 2}

Punch load-displacement curves are shown in Fig. 3. It can be seen that DD3IMP overestimates the load compared both with experimental results and those from Numisheet'99. Whereas with Pam-Stamp, wrinkles developed during the second stage after a displacement of $20 \mathrm{~mm}$. Wrinkles appear when the sheet is under the blank-holder. Decreasing the gap between the die and the blank-holder down to the initial thickness of the blank does not prevent the formation of wrinkles. However, the thickness distribution obtained with Pam-Stamp is close to the average of Numisheet results. Thicknesses obtained with DD3IMP are, as for the first stage, closer to the experimental ones. The tendency of overestimating the thickness at $90^{\circ}$ to the RD remains. Evolution of the blank-holder force during the second stage (Fig. 9) confirms that with Pam-Stamp the blank-holder does not play its role.

\subsection{Occurrence of strain path changes}

In two numerical studies [2,6], for large drawing ratios (1.87 and 1.43 for the first and second stages, respectively [2] - 1.5 for both stages [6]), authors reported the development of necking near the cup bottom in the second stage, when using an isotropic hardening model. This necking is not observed in the case of the direct re-drawing [6], nor when a kinematic hardening model is used instead of an isotropic one [2]. This suggests the occurrence of strain path changes during the forming process. For example, a material point initially located in the outer part of the blank will undergo circumferential compression and radial elongation then a bending and unbending and eventually a plane strain 

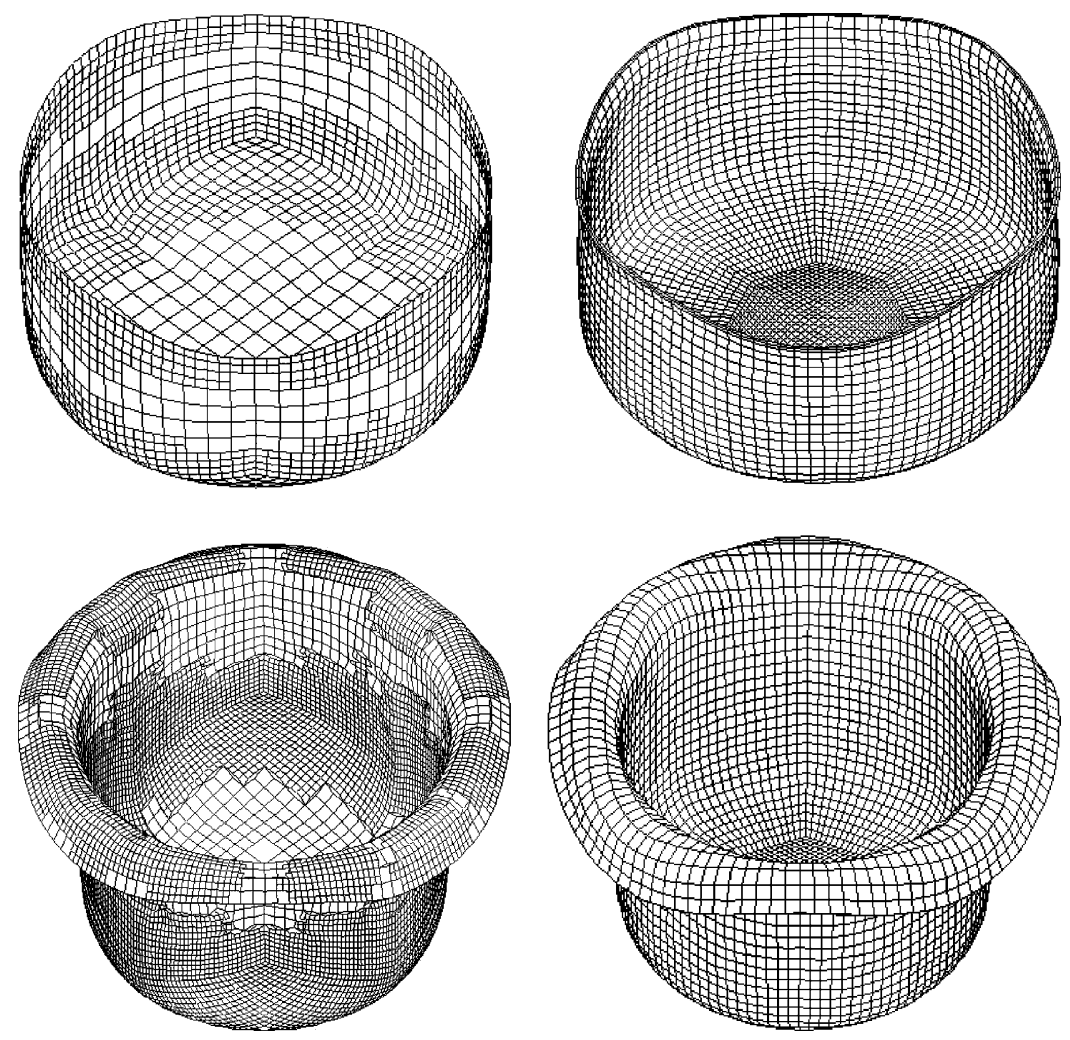

Fig. 9. Evolution of the blank-holder force as a function of the punch stroke.

tension in the cup wall. The amplitude of such changes of the strain rate tensor direction can be estimated with the parameter $\beta$ defined by: $\beta=\underline{\mathbf{A}}_{1}: \underline{\mathbf{A}}_{2}$, where $\underline{\mathbf{A}}_{i}$ is the normalised tensor colinear with the strain rate tensor at increment $i$ in the strain space [18]. $\beta$ varies continuously from -1 (stress reversal) up to 1 (continuous loading) by going through 0 (orthogonal loading). The material behaviour during the second strain path change depends on the $\beta$-value and

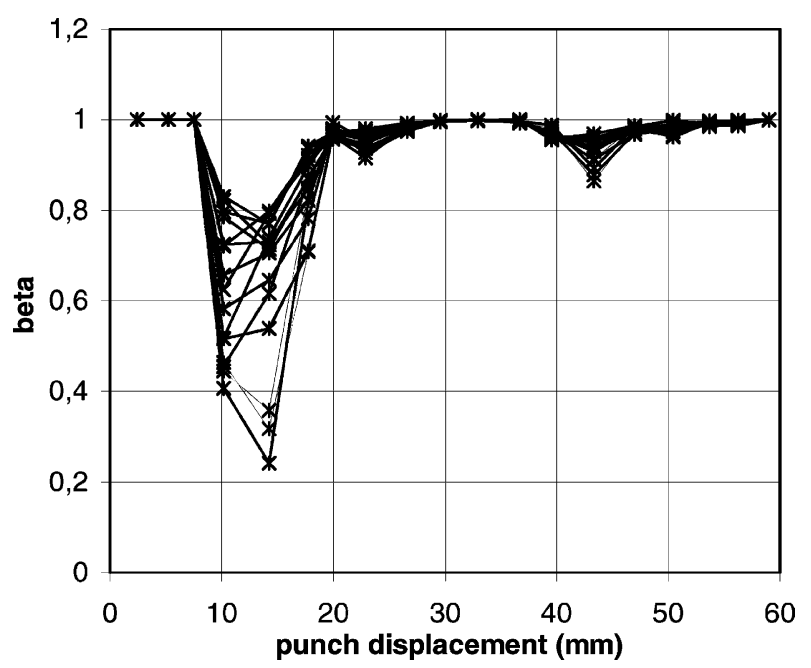

Fig. 10. Evolution of the parameter $\beta$ at a radial distance of $58 \mathrm{~mm}$, at different orientations to the RD, during stage 1 . exhibits a Baushinger effect as well as a plateau on the stress-strain curve $(\beta=-1)$ or a softening $(\beta=0)$.

Calculations of the parameter $\beta$ have been performed with DD3IMP. The incremental plastic strain tensor $\mathrm{d}_{\underline{\varepsilon}}^{\mathrm{P}}$ is given at each increment of the finite element resolution and $\beta$ is calculated in-between two states corresponding to two punch strokes. Strain path changes occur both during the first stage (Fig. 10) and the second one (Fig. 11).

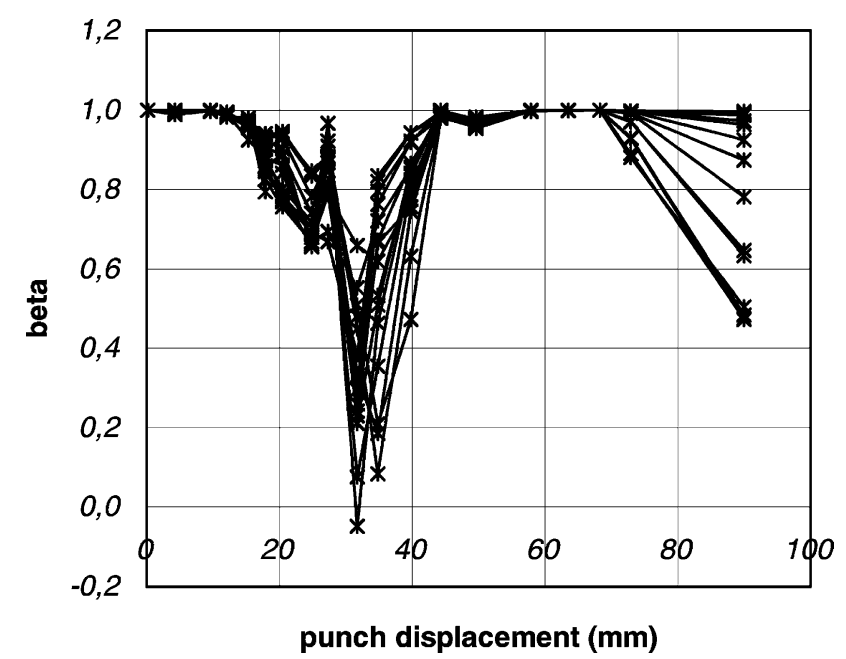

Fig. 11. Evolution of the parameter $\beta$ at a radial distance of $58 \mathrm{~mm}$, at different orientations to the RD, during stage 2 . 
A similar behaviour for all nodes at a given radial distance is noted, but the $\beta$-values depend on the orientation. Values of the order of 0.3-0.5 are encountered, down to values close to 0 . Strain levels attained up to the strain path change as well as the quantitative importance of these changes compared to continuous loading should be further investigated.

\section{Conclusion}

An experimental investigation of the reverse re-drawing of mild steel sheets have been performed. Punch forces have been recorded and the thickness at $0^{\circ}, 45^{\circ}$ and $90^{\circ}$ to the RD measured at the end of the two stages. Numerical simulations were carried out with Pam-Stamp (dynamic explicit with shell elements) and DD3IMP (static implicit with solid elements). The following points should be emphasised:

(1) Experimental and simulated results lie within a range of $20 \%$. The agreement is good in the first stage but problems are encountered for the second one: wrinkle formation with Pam-Stamp and overestimation of the punch force with DD3IMP. Concerning the thickness predictions, they are closer to experiments with DD3IMP. However, only the thickness in the RD is correctly predicted. The thickness is overestimated in the two other directions.

(2) A better prediction of the thickness is expected by improving the description of the anisotropic behaviour, with model parameters identified from both the uniaxial stress-strain curves and the $r$ ratios.

(3) Strain path changes occur during the two stages of the reverse process. This suggests that the isotropic hardening model cannot provide an accurate description of the material behaviour during the forming process.

\section{Acknowledgements}

S. Thuillier is indebted to the Région Bretagne for financial support. The authors are grateful to the Portuguese Foundation for Science and Technology (FCT), who financially supported this work, through the Program POCTI (Portuguese Government and FEDER).

\section{References}

[1] J.C. Gelin, P. Picart (Eds.), Proceedings of the Fourth International Conference and Workshop on Numerical Simulation of 3D Sheet Forming Processes (Benchmarks Numisheet'99), Besançon, France, 1999, pp. 871-932.

[2] L. Cannizzaro, F. Micari, S. Noto La Diega, Finite element analysis of the reverse drawing process, J. Mater. Process. Technol. 24 (1990) $441-450$.

[3] M.A. Ahmetoglu, J. Brandelstein, G.L. Kinzel, T. Altan, Deep drawing and reverse redrawing of aluminum alloy cups, Technical Paper No. MF95-122, NAMRC XXIII, 1995.

[4] D.K. Min, B.H. Jeon, H.J. Kim, N. Kim, A study on process improvements of multi-stage deep-drawing by the finite element method, J. Mater. Process. Technol. 54 (1995) 230-238.

[5] A. Barrata da Rocha, J. Ferreira Duarte, Tecnologia da embutidura, APTCP, 1992.

[6] M.H. Parsa, K. Yamaguchi, N. Takakura, S. Imatani, Consideration of the re-drawing of sheet metals based on finite element simulation, J. Mater. Process. Technol. 47 (1994) 87-101.

[7] A. Col, Consultac, Private communication, 2001

[8] S.K. Esche, M.A. Ahmetoglu, G.L. Kinzel, T. Altan, Numerical and experimental investigation of redrawing of sheet metals, J. Mater. Process. Technol. 98 (2000) 17-24.

[9] J. Danckert, K.B. Nielsen, P. Hojbjerg, Experimental investigation of Numisheet'99 benchmark test C, in: J.C. Gelin, P. Picart (Eds.), Proceeding of the Fourth International Conference and Workshop on Numerical Simulation of 3D Sheet Forming Processes (Numisheet'99), Besançon, France, 1999, pp. 637-642.

[10] L.F. Menezes, C. Teodosiu, Three-dimensional numerical simulation of the deep-drawing process using solid finite element, J. Mater. Process. Technol. 97 (2000) 100-106.

[11] S. Thuillier, P.Y. Manach, Development of a deep drawing device on a tensile test machine, J. Experimental Mechanics, submitted for publication.

[12] R. Hill, Math. Proc. Camb. Philos. Soc. 85 (1979) 179-191.

[13] S. Thuillier, L. Alves, L.F. Menezes, P.Y. Manach, Comparison of experimental and simulated results for a mild steel and a dual-phase steel deformed under tension and deep drawing, in: Proceedings of Materiais 2001, Coimbra, Portugal, April 9-11, 2001, Mater. Sci. Forum, in press.

[14] F. Barlat, D.J. Lege, J.C. Brem, A six-component yield function for anisotropic materials. Int. J. Plast. 7 (1991) 693.

[15] S.P. Wang, S. Choundry, T.B. Wertheimer, Comparison between the static implicit and dynamic explicit methods for FEM simulation of sheet forming processes, in: Proceedings of Numiform 1998, University of Twente, The Netherlands, 1998, pp. 245-250.

[16] J.L. Alves, L.F. Menezes, Application of tri-linear and tri-quadratic 3D solid finite elements in sheet metal forming process simulations, in: K.-I. Mori (Ed.), Simulation of Material Processing: Theory, Methods and Applications, Proceedings of Numiform 2001, pp. 639-644.

[17] Proceedings of the International Conference on FE-simulation of 3D Sheet Metal Forming Processes in Automotive Industry, May 14-16, 1991, Zürich, Switzerland.

[18] E.F. Rauch, J.H. Schmitt, Dislocation substructures in mild steel deformed in simple shear, Mater. Sci. Eng. A 113 (1989) 441-448. 